\title{
BIVENTRICULAR REPAIR FOR AORTIC ATRESIA OR HYPOPLASIA AND VENTRICULAR SEPTAL DEFECT
}

Richard G. Ohye, MD

Koji Kagisaki, MDa

Lisa A. Lee, $\mathrm{MD}^{\mathrm{b}}$

Ralph S. Mosca, MD

Caren S. Goldberg, MD

Edward L. Bove, MD
Objective: Aortic valve atresia or hypoplasia can present with a ventricular septal defect and a normal mitral valve and left ventricle. These patients may be suitable for biventricular repair, although the optimal initial management strategy remains unknown. Methods: From January 1991 through March 1999, 20 patients with aortic atresia or hypoplasia and ventricular septal defect underwent operation with the intent to achieve biventricular repair. Aortic atresia was present in 7 patients, and aortic valve hypoplasia was present in 13 patients. Among those patients with aortic hypoplasia, $Z$-scores of the aortic valve anulus ranged from $\mathbf{- 8 . 8}$ to $\mathbf{- 2 . 7}$. Associated anomalies included interrupted aortic $\operatorname{arch}(n=12$ patients), coarctation ( $n=6$ patients), aortopulmonary window ( $n=1$ patient), and heterotaxia $(n=1$ patient). Nine patients were staged with an initial Norwood procedure followed by biventricular repair in 8 patients. One patient awaits biventricular repair after a Norwood procedure. The conditions of 11 patients were corrected with a single procedure. Results: Among the 9 patients who underwent staged repair, there were no deaths after the Norwood procedure and 1 death after biventricular repair. For the 11 patients who underwent a primary biventricular repair, there was 1 early death and 2 late deaths from noncardiac causes. Follow-up ranged from 1 to 85 months (mean, 28 months). Actuarial survival for the entire group was $\mathbf{7 8 \%} \pm \mathbf{1 0 \%}$ at 5 years and was not significantly different between staged repair $(89 \%)$ and primary biventricular repair $(73 \%)$. Conclusions: Both primary and staged biventricular repair for patients with aortic atresia or hypoplasia and ventricular septal defect may be performed with good late survival. Refinements in technique of conduit insertion and arch reconstruction have resulted in primary biventricular repair becoming our preferred approach. (J Thorac Cardiovasc Surg 1999;118:648-54)
A tresia or hypoplasia of the aortic valve is generally associated with a hypoplastic left ventricle and mitral valve. As part of the hypoplastic left heart syndrome, this condition is therefore unsuitable for biven-

From the Division of Pediatric Cardiovascular Surgery, Section of Cardiac Surgery, a and the Division of Pediatric Cardiology, Department of Pediatrics, ${ }^{\text {b }}$ C. S. Mott Children's Hospital, University of Michigan School of Medicine, Ann Arbor, Mich.

Read at the Seventy-ninth Annual Meeting of The American Association for Thoracic Surgery, New Orleans, La, April 18-21, 1999.

Received for publication April 22, 1999; revisions requested May 6, 1999; revisions received May 13, 1999; accepted for publication May 18, 1999.

Address for reprints: Edward L. Bove, MD, Pediatric Cardiovascular Surgery, F7830 Mott Hospital, 1500 East Medical Center Dr, Ann Arbor, MI 48109.

Copyright (C) 1999 by Mosby, Inc.

$0022-5223 / 99 \$ 8.00+0 \quad \mathbf{1 2 / 6 / 1 0 0 0 8 2}$ tricular repair (BVR). However, in $4 \%$ to $7 \%$ of patients with aortic atresia, a ventricular septal defect (VSD) coexists and may allow for the development of a normal mitral apparatus and left ventricle. ${ }^{1-4}$ Additionally, even when the aortic valve remains patent, it may be of insufficient size to permit standard BVR without subsequent left ventricular outflow tract obstruction after correction of associated defects. Although the ultimate goal is to achieve a BVR when the left ventricle and mitral valve are of adequate size, the optimal initial management strategies remain unclear and only sporadic case reports of 1- or 2-stage BVR have appeared in the literature. ${ }^{5-10}$

This report summarizes our experience with patients who underwent BVR for aortic atresia or hypoplasia to assess early and intermediate-term outcome, strategies of initial surgical approach, and the techniques of achieving BVR. 


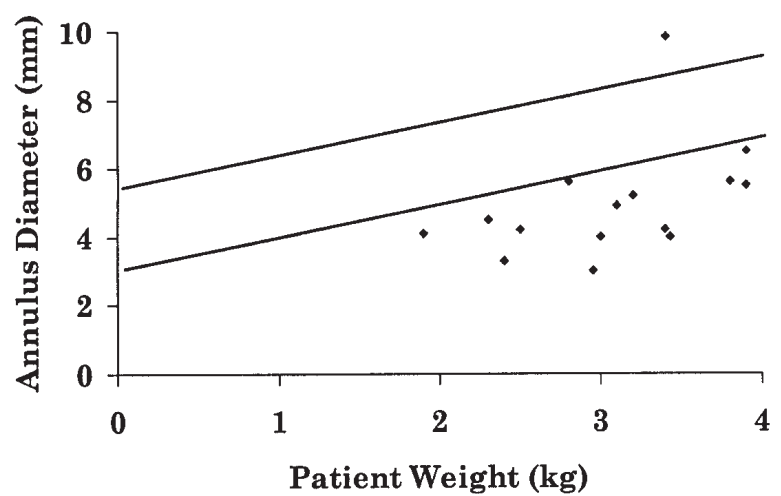

Fig 1. Aortic anulus measurements. Lines represent 2 SDs above and below the mean. The single patient above 2 SDs from the mean had no forward flow across the aortic valve.

Table I. Associated anomalies

\begin{tabular}{lr}
\hline Anomaly & $n$ \\
\hline Cardiac & 12 \\
Interrupted aortic arch & 6 \\
Coarctation of the aorta & 1 \\
Aortopulmonary window & 1 \\
$\quad$ Heterotaxia syndrome with dextrocardia & \\
Noncardiac & 6 \\
DeGeorge syndrome & 2 \\
CHARGE association & 1 \\
Holt-Oram syndrome & \\
\hline
\end{tabular}

\section{Patients and methods}

From January 1991 through March 1999, 20 patients with aortic atresia or significant aortic valve hypoplasia in association with a VSD and a normal mitral valve and left ventricle were seen at C. S. Mott Children's Hospital, the University of Michigan Health System. All patients were treated with the intent to perform BVR by either a staged technique consisting of an initial Norwood procedure followed by BVR ( $\mathrm{n}=9$ patients) or by a primary BVR $(\mathrm{n}=11$ patients). There were 15 male $(75 \%)$ and 5 female $(25 \%)$ patients. At the time of initial operation, the patients ranged in age from 2 to 58 days (mean, 18 days) and in weight from 1.9 to $4.6 \mathrm{~kg}$ (mean, $3.1 \mathrm{~kg}$ ).

There were 7 patients with aortic atresia $(35 \%)$ and 13 patients with aortic hypoplasia $(65 \%)$. For the 13 patients with aortic hypoplasia, the diameter of the aortic anulus was measured by echocardiography. All patients except 1 had an anulus diameter less than 2 standard deviations below the mean (Fig 1). The 1 exception, however, had no forward flow across the aortic valve as the result of a mass that was present within the aortic anulus and was therefore functionally atretic. Excluding this patient, the Z-scores ranged from -2.7 to -8.9 , and the absolute diameters ranged from 3 to $5.5 \mathrm{~mm}$.

Adequacy of the left ventricle and mitral apparatus was evaluated by complete preoperative Doppler echocardiogra-

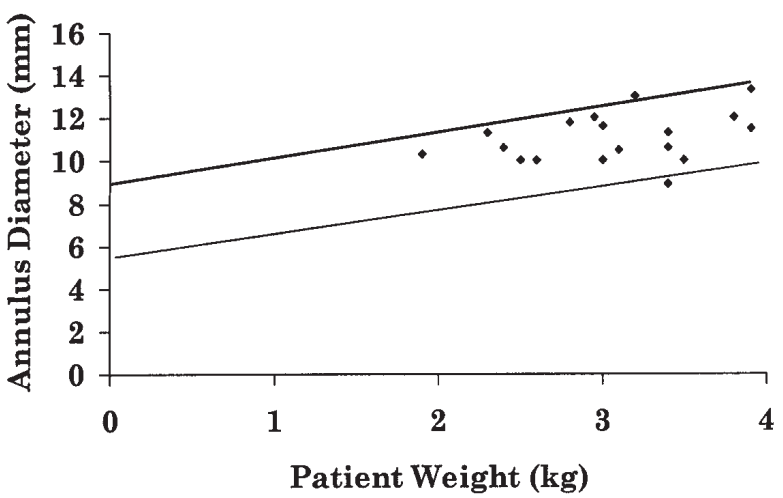

Fig 2. Mitral anulus measurements. Lines represent 2 SDs above and below the mean.

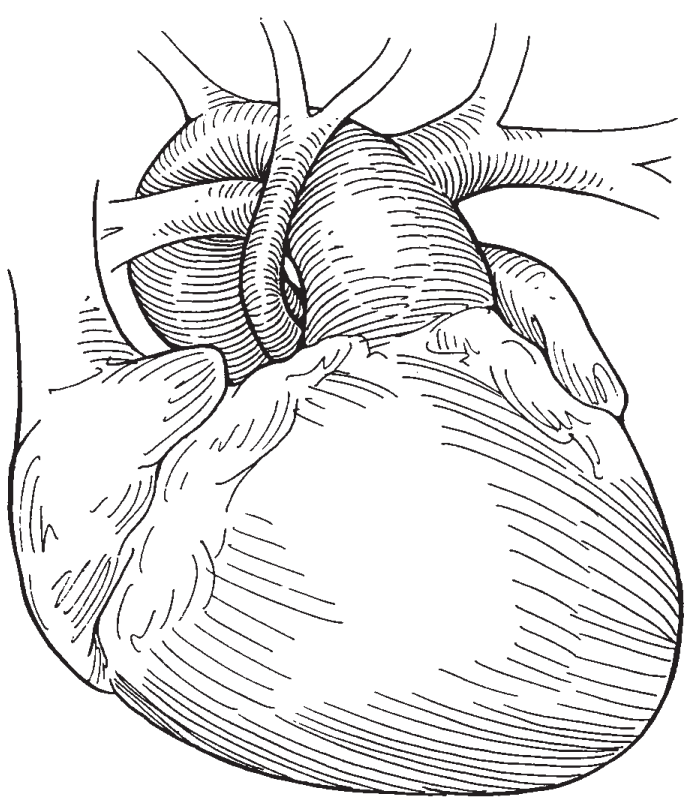

Fig 3. Patient 1. Preoperative anatomy shows aortic valve and ascending aorta hypoplasia and interrupted right aortic arch. The carotid arteries are branches of the proximal aorta, although the right subclavian artery is a branch of the rightsided descending aorta and the left subclavian artery is a branch of the left pulmonary artery.

phy in all patients. In each patient, the left ventricle was apex forming with good function, and there was no indication of endocardial fibroelastosis. In 18 patients, echocardiographic measurements of mitral valve anulus were available for analysis. Of the 18 patients, 17 patients fell within 2 standard deviations of the mean (Fig 2).

Associated lesions were common findings in this group of patients. There were 12 patients with interrupted aortic arch, 6 patients with significant coarctation of the aorta, 1 patient 


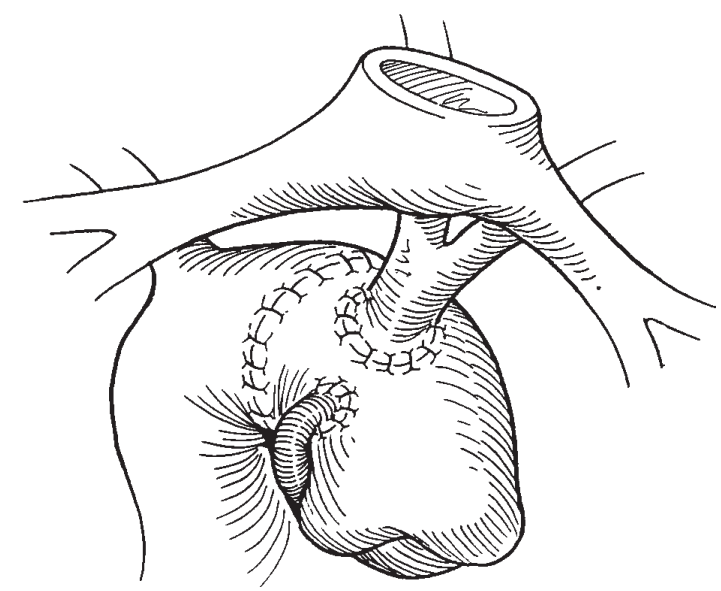

Fig 4. Patient 1 . The main pulmonary artery was divided, and the branch pulmonary arteries were relocated anteriorly. The distal aorta was anastomosed directly to the main pulmonary artery. The hypoplastic ascending aorta was transected, and each end was sewn end-to-side to the main pulmonary artery.

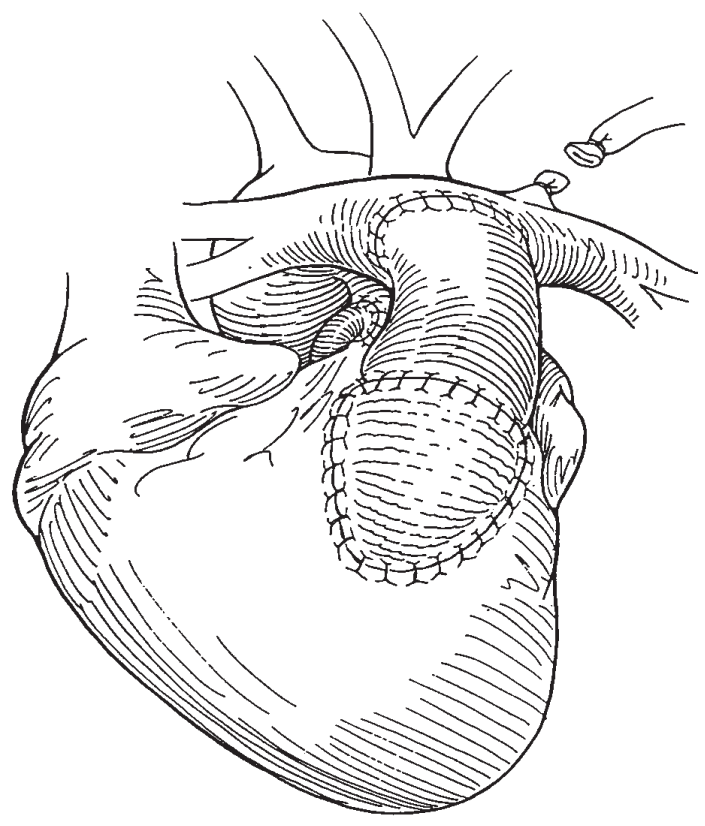

Fig 5. Patient 1. After the left ventricular output was baffled through the VSD to the pulmonary valve and the left subclavian artery was divided, right ventricle to pulmonary artery continuity was re-established with a homograft. The anterior location of the branch pulmonary arteries facilitated placement of the conduit.

with an aortopulmonary window, and 1 patient with heterotaxia syndrome and dextrocardia. Important noncardiac conditions included DiGeorge syndrome in 6 patients, CHARGE association in 2 patients, and Holt-Oram syndrome in 1 patient (Table I).

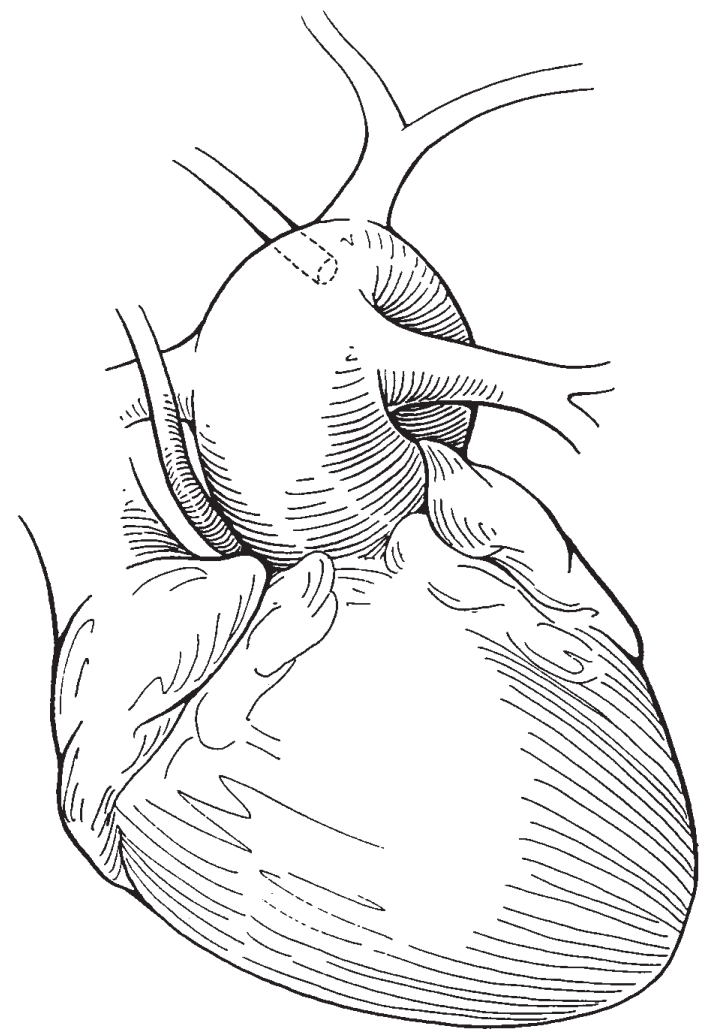

Fig 6. Patient 2. Preoperative anatomy shows aortic atresia and type- $\mathrm{C}$ interruption of the aortic arch. The coronaries are supplied retrograde from the right carotid artery. The left carotid and subclavian arteries arise from a common trunk off the descending aorta, as does an aberrant right subclavian artery.

Operative techniques. Staged repair was accomplished by an initial Norwood procedure by standard technique. ${ }^{11}$ This procedure was followed 6 to 12 months later with a BVR. Atrial septectomy was not performed in the presence of a restrictive atrial defect. Single-stage repair generally involved a combination of Norwood and Rastelli techniques. Arch interruption was repaired by direct anastomosis, with the repair tailored to the individual anatomy. The pulmonary artery was anastomosed to the augmented aorta, and a right ventriculotomy was performed to provide access to the VSD. It was necessary to enlarge the VSD in 15 of 19 of the patients (79\%) to insure an unobstructed left ventricular outflow tract. A prosthetic patch was then placed to connect the left ventricle to the pulmonary valve. Right ventricle to pulmonary artery continuity was established with a cryopreserved homograft pulmonary valve conduit. Because of the wide individual variation in anatomy, a variety of techniques were used for arch reconstruction and pulmonary artery anastomosis, as illustrated in the following 2 patients.

Clinical summaries. The first patient was born prematurely at 32 weeks of gestation with a birth weight of $2.0 \mathrm{~kg}$. His anatomy consisted of aortic hypoplasia ( $\mathrm{Z}$ score, -4.2$)$ with 


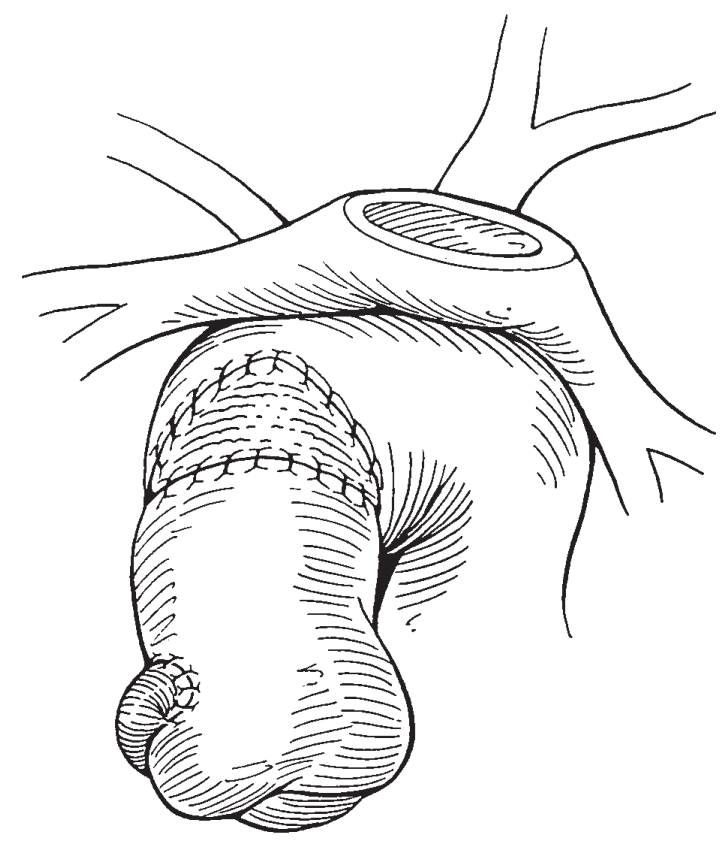

Fig 7. Patient 2. After the branch pulmonary arteries were relocated anteriorly, the distal aorta was anastomosed directly to the main pulmonary artery. An autologous pericardial patch was used on the anterior aspect to insure a tension-free connection. The proximal aorta was sewn end-to-side to the main pulmonary artery.

an outlet VSD. There was an associated interrupted rightsided aortic arch, and the ascending aorta divided into right and left common carotid arteries. The right subclavian artery was a branch of the descending right-sided aorta, and the left subclavian artery arose from the left pulmonary artery (Fig 3). He underwent BVR on day 12 of life. The main pulmonary artery was divided proximal to the branch pulmonary arteries; the ductus arteriosus was resected, and the distal aorta was anastomosed directly to the main pulmonary artery. The branch pulmonary arteries were relocated anterior to the aorta by way of a LeCompte maneuver. ${ }^{12}$ The hypoplastic ascending aorta was divided, and each end was anastomosed end-to-side into the main pulmonary trunk to supply the coronary and carotid arteries (Fig 4). A right ventriculotomy was performed, and the VSD was enlarged anteriorly and superiorly. A polytetrafluoroethylene patch was placed to direct the left ventricular output to the pulmonary valve. After the left subclavian artery was divided, the branch pulmonary arteries were anastomosed to a cryopreserved pulmonary homograft valve conduit. This anastomosis was facilitated by the anterior position of the distal pulmonary arteries (Fig 5).

The second patient was a $2.6 \mathrm{~kg}$ premature neonate with aortic atresia, VSD, and an associated interrupted aortic arch (type C). The ascending aorta was extremely diminutive in size $(1.5 \mathrm{~mm})$ and continued as a right carotid artery only. Retrograde flow through the right carotid artery supplied

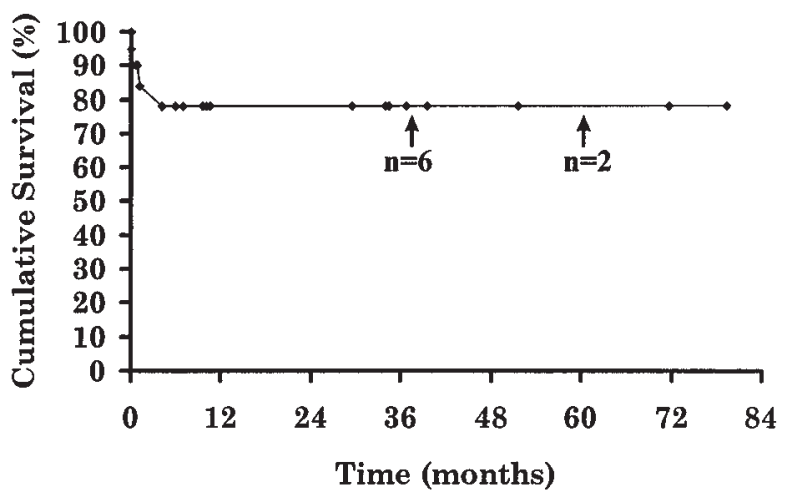

Fig 8. Five-year actuarial survival for the entire group of 20 patients. The 5-year actuarial survival of $78 \%$ is encouraging, albeit based on a limited number of long-term survivors (at 3 years, 6 survivors; at 5 years, 2 survivors).

blood to the coronary arteries as demonstrated by Doppler and magnetic resonance imaging studies. The left carotid and left subclavian arteries originated as a common trunk from the descending aorta, as did an aberrant right subclavian artery (Fig 6). He underwent primary BVR on day 47 of life. As in the first case report, the main pulmonary artery was divided, and the ductal tissue was resected. The branch pulmonary arteries were relocated anterior to the aorta. The descending aorta and main pulmonary trunk were anastomosed directly, with an autologous pericardial patch inserted anteriorly to insure a tension-free anastomosis (Fig 7). A polytetrafluoroethylene patch was used to channel the left ventricle to the pulmonary valve after enlarging the VSD, and right ventricle to pulmonary artery continuity was accomplished with a cryopreserved pulmonary homograft positioned anterior to the reconstructed aorta.

\section{Results}

Actuarial survival for the entire group of 20 patients was $78 \% \pm 10 \%$ (16/20 patients) at 5 years (Fig 8$)$. The mean follow-up was 28 months and ranged from 1 to 59 months. Among the patients who underwent staged repair, survival was 8 of 9 patients $(89 \%)$. There were no deaths after the Norwood procedure and 1 death from sepsis and low cardiac output after BVR. One patient has not yet undergone BVR. Among the patients who underwent primary BVR, survival was 8 of 11 patients $(73 \%)$, which was not significantly different when compared with the staged-repair group. One early death occurred from low cardiac output. There were 2 additional late deaths, both from noncardiac causes. The first death occurred in a premature infant 4 months after successful repair from respiratory insufficiency as the result of bronchopulmonary dysplasia. The second patient died 34 days after successful repair from docu- 
mented graft-versus-host disease associated with DiGeorge syndrome.

Significant morbidity included 3 episodes of sepsis. There were no instances of complete heart block, and 1 patient with sick sinus syndrome required permanent pacemaker placement. Three patients underwent reoperation related to their repair. One patient required intervention for closure of a hemodynamically significant residual VSD, and 1 patient required augmentation of branch pulmonary artery stenosis. One patient experienced both a partial VSD patch dehiscence and branch pulmonary artery stenosis that required surgical intervention. In addition, 1 patient underwent diaphragmatic plication for a paralyzed hemidiaphragm. Right ventricle to pulmonary artery conduit replacement has been required in 3 patients at age 2, 3, and 4 years. No patient has required reoperation for left ventricular outflow tract obstruction.

Complete Doppler echocardiographic evaluation was performed on all patients before discharge. No patient had either left or right ventricular outflow tract obstruction. There were 3 instances of mild-to-moderate branch pulmonary artery stenosis, 1 of which required surgical correction 7 weeks after the initial operation. One residual VSD requiring reoperation was identified. Valvular abnormalities included mild tricuspid regurgitation in 2 patients and mild neoaortic insufficiency in 1 patient.

\section{Discussion}

Although generally associated with a hypoplastic left ventricle, aortic atresia can occasionally be found with a VSD and a normal mitral valve and left ventricle. These patients account for $4 \%$ to $7 \%$ of the cases of aortic atresia and are candidates for BVR. ${ }^{1-3}$ In these patients, the VSD provides an "outlet" for the developing left ventricle and mitral valve in utero. The maintenance of flow across the mitral valve and through the left ventricle allows for their normal growth and development. When the aortic valve is patent but small, the hypoplasia of the aortic valve anulus prevents simple operative or balloon valvuloplasty without significant residual left ventricular outflow tract obstruction. In addition, the high incidence of associated interrupted aortic arch (60\%) and aortic coarctation (30\%) further complicates the repair. In our institution, an aortic valve anulus $\mathrm{z}$-value of -2 or less ( $<95 \%$ of the mean) is considered indicative of a high risk for important residual left ventricular outflow tract obstruction after traditional methods of repair.

Bernhard and colleagues ${ }^{13}$ published a report in 1975 about the use of a ventriculoaortic conduit to alleviate critical congenital aortic stenosis. Freedom and col- leagues ${ }^{1}$ proposed the potential for a BVR in their review of 2 cases of aortic atresia with normal left ventricle. They postulated a procedure that would include baffling of the left ventricle output through the VSD to the pulmonary artery, maintaining aortic communication through the ductus arteriosus and a Potts shunt. Right ventricle to pulmonary artery continuity would then be established with a prosthetic conduit. Norwood and Stellin ${ }^{5}$ published the first case report of BVR in a patient with aortic atresia and an adequate left ventricle in 1981. They performed a 2-stage procedure that involved the placement of a Hancock valved conduit from the left ventricle apex to the descending aorta on day 3 of life, followed by VSD closure at 1 month. In another report, a 2-stage approach was performed in which an initial pulmonary artery banding and conduit placement from the main pulmonary artery to the aorta was followed by the placement of a left ventricle to descending aorta composite graft and VSD closure. ${ }^{6}$ The first report of primary BVR appeared in 1989. ${ }^{7}$ The technique involved the establishment of main pulmonary artery to aorta communication with a homograft and baffling of the VSD to this neoaorta. Right ventricle to distal pulmonary artery continuity was then provided by another homograft conduit.

In this retrospective series, we reviewed our experience, in part, in an effort to define the optimal initial operative approach. The patients in this report underwent BVR either as a single procedure or as a staged repair. The initial surgical approach was generally selected according to patient condition and individual surgeon preference and was not protocol driven. Each technique has inherent strengths and weaknesses. An initial Norwood procedure as part of a staged repair is of "lesser" magnitude than a primary BVR. It avoids the need for a ventriculotomy and placement of a right ventricle-pulmonary artery conduit in these small neonates. However, the postoperative course may be more labile because of the shunt-dependent pulmonary blood flow associated with the Norwood procedure. Although it avoids the shunt-dependent physiologic procedures, the primary BVR is a more complicated operation. It requires a right ventriculotomy and the use of a right ventricle-pulmonary artery conduit.

The technical aspects of BVR present a considerable challenge, including the placement of the right ventricle-pulmonary artery conduit. The course for the conduit is unfavorable because of the long distance from the anteriorly located right ventricle, over the large pulmonary valve, to the posterior pulmonary arteries. An alternative approach involves translocating the branch pulmonary arteries anterior to the neoaorta by a 
LeCompte maneuver, which facilitates the placement of the conduit. Both the ascending aorta and main pulmonary artery are divided, and the distal aorta is anastomosed directly to the main pulmonary artery after the branch pulmonary arteries are translocated anteriorly. The proximal ascending aorta is then reimplanted into the neoaorta to supply coronary blood flow. Alternatively, conduit insertion may be avoided entirely, and a direct anastomosis with or without a monocusp valve may be performed.

In our series, a clear superiority of staged versus primary BVR was not demonstrated. The actuarial survival at 5 years for the entire group was $78 \%(16 / 20$ patients). Survival after primary BVR was $73 \%(8 / 11$ patients) and was not significantly different when compared with $89 \%$ (8/9 patients) for staged repair with initial Norwood followed by BVR at a later date. The deaths in the primary BVR group were influenced by associated conditions. In fact, those patients with significant noncardiac lesions and those patients in poor preoperative condition were usually selected for primary BVR because of concerns regarding their ability to survive shunt-dependent pulmonary blood flow after the Norwood procedure.

Early in our experience, a staged approach for the repair of aortic atresia or hypoplasia with VSD with an initial Norwood procedure followed by BVR was frequently used. This approach was selected, in part, because of the technical difficulties encountered with the placement of the conduit from the anterior right ventricle, over the large reconstructed neoaorta, to the posterior pulmonary arteries. However as our experience evolved, several patients who were in poor clinical condition underwent primary BVR because of concerns regarding their ability to tolerate shunt-dependent pulmonary blood flow after a Norwood procedure. The excellent outcomes of these patients and the use of the LeCompte maneuver, in addition to direct main pulmonary artery to aortic arch anastomosis, facilitated conduit placement and made primary BVR an attractive alternative to a staged approach. Although our data support the conclusion that BVR for aortic atresia or hypoplasia with VSD can be performed by either method with low morbidity and excellent survival, refinements in the technique of conduit insertion and arch reconstruction have resulted in primary BVR becoming our preferred approach.

\section{REFERENCES}

1. Freedom M, Dische MR, Rowe RD. Conal anatomy in aortic atresia, ventricular septal defect, and normally developed left ventricle. Am Heart J 1997;94:689-98.
2. Roberts WC, Perry LW, Chandra RS, et al. Aortic valve atresia: a new classification based on necropsy study of 73 cases. Am J Cardiol 1976;37:753-6.

3. Mahowald JM, Lucas RV, Edwards JE. Aortic valvular atresia: associated cardiovascular anomalies. Pediatr Cardiol 1982;2:99-105.

4. Thiene G, Gallucci V, Macartney FJ, et al. Anatomy of aortic atresia: cases presenting with a ventricular septal defect. Circulation 1979;59:173-8.

5. Norwood WI, Stellin GJ. Aortic atresia with interrupted aortic arch: reparative operation. J Thorac Cardiovasc Surg 1981;81: 239-44.

6. Duffy CE, Muster AJ, DeLeon SY, et al. Successful surgical repair of aortic atresia associated with normal left ventricle. J Am Coll Cardiol 1983;1:1503-6.

7. Austin EH, Jonas RA, Mayer JE, Castaneda AR. Aortic atresia with normal left ventricle: single stage repair in the neonate. $J$ Thorac Cardiovasc Surg 1989;97:392-5.

8. Serraf A, Bruniaux J, Lebidois J, et al. Aortic atresia with normal left ventricle. Ann Thorac Surg 1991;51:1017-9.

9. Bogers AJJC, Sreeram N, Hess J, et al. Aortic atresia with normal left ventricle: one-stage repair in early infancy. Ann Thorac Surg 1991;51:312-4.

10. Francois K, Dollery C, Elliott MJ. Aortic atresia with ventricular septal defect and normal left ventricle: one-stage repair in the neonate. Ann Thorac Surg 1994;58:878-80.

11. Pigott JD, Murphy JD, Barber G, Norwood WI. Palliative reconstructive surgery for hypoplastic left heart syndrome. Ann Thorac Surg 1988;45:122-8.

12. LeCompte Y, Zannini L, Hazan E, et al. Anatomic correction of transposition of the great arteries. J Thorac Cardiovasc Surg 1981;82:629-31

13. Bernhard WF, Poirier V, LaFarge LG. Relief of congenital obstruction to left ventricular outflow with a ventriculo-aortic prosthesis. J Thorac Cardiovasc Surg 1975;69:223-9.

\section{Discussion}

Dr Richard A. Jonas (Boston, Mass). In 1989, Dr Erle Austin reported from our group the first experience with primary 1-stage repair of aortic atresia with a normal left ventricle. There were 4 patients in his initial report. The only patient who died was a patient in whom dextrocardia complicated the reconstruction. We concluded from this initial report that there was no reason that patients with this anomaly should not undergo a primary repair in the neonatal period. This recommendation was consistent with our long-standing philosophy that the earlier one can achieve a normal in-series 2-ventricle circulation, the more probable there will be normal cardiac, pulmonary, brain, and whole body development.

Dr Bove's group has a well-deserved reputation as 1 of the leading centers for infant and neonatal corrective operation. And so I am somewhat confused regarding the conclusion that the authors have drawn from this experience. In one half of their patients, they performed a palliative procedure with a shunt and in one half they undertook a BVR. This means that the patients who underwent palliation had to endure a period of abnormal volume loading as well as the additional costs (financial, emotional, and psychologic) for the family of an early reoperation. I think there is good evidence, such as was 
presented at this meeting yesterday when we described our experience with truncus arteriosus over the last 7 years, that a right ventriculotomy and placement of a right ventricle-pulmonary artery homograft conduit is well tolerated in the neonatal population. The mortality rate, as you heard yesterday in the truncus series, was an acceptable $4 \%$ in spite of the fact that that series included patients with interrupted aortic arch and 4 patients with severe truncal valve regurgitation.

Why have you had any difficulty deciding that a BVR is the preferable option when there is no measurable difference as determined by early mortality and morbidity rates between a 1-stage approach versus a 2-stage approach?

Regarding your experience with DiGeorge syndrome in a patient with hypoplastic left heart syndrome, which is an unusual association in our experience, you noted that a patient died from what was determined to be graft-versushost reaction with a blood transfusion. I have to say that my own impression has been that immunologic problems in children who are labeled as having DiGeorge syndrome has been, in fact, not only rare, but as far as I am aware, in our experience, has not occurred. I would like to hear some more specific details as to how this was determined to be graft-versushost and how this manifested itself with a blood transfusion.

Dr Ohye. In response to your first question regarding the choice of a staged approach, I think I would have to agree with you. I will talk a little bit about the evolution of how we came to primary BVR. Initially, we were concerned in these small neonates with the placement of a right ventricle-pulmonary artery conduit from the anterior right ventricle over an augmented aorta and then to a posteriorly placed pulmonary artery. As our experience grew, we began translocating the branch pulmonaries anteriorly. As we began to see that the patients in the primary repair group were doing just as well, it has been our bias more recently to perform a complete repair in 1 stage.

In regard to the patient with DiGeorge syndrome, this is the first case that I am aware of also. The patient was diagnosed by a skin biopsy. He displayed typical lesions of graft-versushost disease, and a skin biopsy confirmed the diagnosis.

Dr Christo I. Tchervenkov (Montreal, Quebec, Canada). I would like to raise a philosophic issue that was briefly dis- cussed in the postgraduate course, the question of univentricular versus BVR or staged versus single-stage approach for similar groups of patients. It seems to me that we should exhibit the same perseverance toward the biventricular approach in the borderline patient as we exhibited with the Norwood operation for the hypoplastic left heart syndrome.

I also support the comments made by Richard Jonas in favor of the biventricular approach. We have in the past used multiple arguments against palliation for many other lesions in favor of early primary repair for even complex lesions in the neonatal and the infant periods. The logic escapes me somewhat as to why you have used a very complex reconstruction based on a single ventricle physiology for only 6 months, after which you again undertook the relatively complex intracardiac BVR.

Last year at the annual meeting of The American Association for Thoracic Surgery, we presented our results of single-stage BVR with concomitant aortic arch reconstruction in 40 consecutive patients with a variety of complex cardiac defects, including patients similar to your series. Our perioperative mortality rate was only $5 \%$, with the 2 deaths occurring in patients with significant left ventricular hypoplasia.

Were you able to sequentially analyze and index the body surface area, the size of the aortic valve anulus, the left ventricular outflow tract, and the size of the VSD as these patients have progressed through your management strategies in both groups? How did the size of these structures change over time, and how did it influence your decision-making process?

Dr Ohye. Again, I would reiterate that we would also support that concept where BVR is preferred over univentricular repair. All 20 patients were seen with the intent to perform BVR and, with the exception of 1 patient who awaits BVR, have undergone 2-ventricle repair.

In regard to the VSD, we did not do any specific measurements or index it to body surface area before or during the operation to help our operative planning. We did, however, enlarge the VSD in $80 \%$ of our patients. As a rough rule of thumb, we make it at least the size of the aortic anulus.

We had no cases of left ventricular outflow tract obstruction either in the early or late postoperative period. 